\title{
Output-Feedback Controller Based Projective Lag-Synchronization of Uncertain Chaotic Systems in the Presence of Input Nonlinearities
}

\author{
Abdesselem Boulkroune, ${ }^{1}$ Sarah Hamel, ${ }^{1}$ Farouk Zouari, ${ }^{2}$ \\ Abdelkrim Boukabou, ${ }^{3}$ and Asier Ibeas ${ }^{4,5}$ \\ ${ }^{1}$ LAJ, Automatic Control Department, University of Jijel, BP 98, Ouled-Aissa, 18000 Jijel, Algeria \\ ${ }^{2}$ LARA, École Nationale d'Ingénieurs de Tunis, Université de Tunis El Manar, BP 37, Le Belvédère, 1002 Tunis, Tunisia \\ ${ }^{3}$ Department of Electronics, University of Jijel, BP 98, Ouled-Aissa, 18000 Jijel, Algeria \\ ${ }^{4}$ Department of Telecommunications and Systems Engineering, Universitat Autònoma de Barcelona, Bellaterra, 08193 Barcelona, Spain \\ ${ }^{5}$ Departamento de Ingeniería, Facultad de Ciencias Naturales e Ingeniería, Universidad de Bogotá Jorge Tadeo Lozano, \\ 22 Street, No. 4-96, Mod. 7A, Bogotá 110311, Colombia
}

Correspondence should be addressed to Abdesselem Boulkroune; boulkroune2002@yahoo.fr

Received 20 December 2016; Revised 6 February 2017; Accepted 12 February 2017; Published 19 March 2017

Academic Editor: Tomasz Kapitaniak

Copyright (C) 2017 Abdesselem Boulkroune et al. This is an open access article distributed under the Creative Commons Attribution License, which permits unrestricted use, distribution, and reproduction in any medium, provided the original work is properly cited.

This paper solves the problem of projective lag-synchronization based on output-feedback control for chaotic drive-response systems with input dead-zone and sector nonlinearities. This class of the drive-response systems is assumed in Brunovsky form but with unavailable states and unknown dynamics. To effectively deal with both dead-zone and sector nonlinearities, the proposed controller is designed in a variable-structure framework. To online learn the uncertain dynamics, adaptive fuzzy systems are used. And to estimate the unavailable states, a simple synchronization error is constructed. To prove the stability of the overall closedloop system (controller, observer, and drive-response system) and to design the adaptation laws, a Lyapunov theory and strictly positive real (SPR) approach are exploited. Finally, three academic examples are given to show the effectiveness of this proposed lag-synchronization scheme.

\section{Introduction}

The chaos synchronization has attracted great attention and has been extensively studied [1-14], since it was suggested originally by Pecora and Carroll in [15]. The basic configuration of chaos synchronization consists of two chaotic systems: a drive (master) system and a response (slave) system. These systems can be identical but with different initial conditions (IC) or quite different. The response system is driven via some transmitted (drive) signals so that the trajectories of the response system synchronize with that of the drive system.

In the literature, there are many types of the chaos synchronization such as complete synchronization (CS) $[1,2]$, generalized synchronization (GS) $[3,4]$, projective synchronization (PS) [5, 6], and lag-synchronization (LS) [7]. In PS, the state vectors of two synchronized systems evolve in a proportional scale. In LS, due to signal propagation delays in the environment, it is reasonable to require the response system at time $(t)$ to synchronize the drive one at time $(t-\tau)$, where $\tau$ is the propagation delay (lag) [16]. In recent years, lag-synchronization has attracted a great deal of attention. Some results have been reported about LS [8-14, 16-18]. Besides, over the past 25 years, a variety of methods have been proposed for chaos synchronization, such as sliding mode control [19,20], active control [21, 22], adaptive control $[23,24]$, and fuzzy control [25-27] which are designed via the universal approximation theorem [28].

In real applications of chaos synchronization, the state vectors of drive-response systems are not available for measurement, except the outputs of drive-response systems. Thus, designing a synchronization scheme based on an outputfeedback controller (i.e., an observer-based controller) is 
required. Based on state observer, some adaptive control systems were designed in [29-32]. These systems involve strictly positive real (SPR) concept on the observation-error dynamics. The dynamics of the observation errors, which are originally not SPR, are augmented by an appropriate low-pass filter designed to meet the SPR concept.

On the other hand, most of the above works are only valid for chaotic systems without dynamical disturbances and input nonlinearity. However, in practice, the chaotic systems are inevitably affected by uncertain dynamical disturbances. The existence of these disturbances can generally lead to the synchronization failure and cause undesirable results. How to enhance the disturbance compensation or attenuation is of great significance $[33,34]$. Besides, owing to the physical limitations, the practical implementations of the control systems are frequently exposed to input nonlinearities (backlash, dead-zone, and saturation). It has been shown that these input nonlinearities can cause a serious degradation of the system performances and in a worst-case system failure. So, the design of a controller for chaos synchronization by considering of the external disturbances and input nonlinearities is of significant importance [31-39]. To effectively deal with these problems, the control schemes have been generally designed in a variable-structure control framework.

Motivated by the above discussions, in this paper, we aim at addressing the problem of projective lag-synchronization for a class of uncertain chaotic systems subject to uncertain external dynamical disturbances and input nonlinearities (sector nonlinearities with dead-zone). This synchronization can be realized through an appropriate fuzzy adaptive variable-structure controller based on a state observer. Compared with the previous works on the chaos synchronization and control [8-14, 16-20, 31-39], the main contributions of this paper are the following:

(i) A novel projective lag-synchronization system based on fuzzy adaptive variable- structure output-feedback control is designed for unknown perturbed chaotic systems containing dead-zone nonlinearity.

(ii) The model of the chaotic drive-response system is assumed to be completely different, unknown (except its relative degree), subject to dynamical disturbances, with input dead-zone and sector nonlinearities, and immeasurable states. Besides, its dynamics should not satisfy the SPR property. To authors' best knowledge, such a class of chaotic (drive-response) systems with all these properties has not been previously considered in the open synchronization literature.

(iii) Unlike in [40-46], by using the SPR property together with Lyapunov theory, the stability of the resultant closed-loop system is carefully established. Recall that many previous works requiring the SPR property, for example, [40-46], have not been derived rigorously in mathematics, as stated in [47].

(iv) By designing a linear observer to estimate the lag-synchronization errors, only the outputs of the response-drive system are assumed to be measurable in this synchronization scheme. (v) The designed fuzzy adaptive control is very simple and has only two adaptive parameters. So, this controller is of practical significant importance.

\section{System Description and Problem Formulation}

Consider the following class of drive-response chaotic systems:

$$
\begin{aligned}
& y_{x}^{(n)}=F_{d}(x)+D_{d}(t, x) \\
& y_{z}^{(n)}=F_{r}(z)+u+D_{r}(t, z)
\end{aligned}
$$

or equivalently of the form

$$
\begin{aligned}
& \dot{x}=A x+B\left[F_{d}(x)+D_{d}(t, x)\right] \\
& \dot{z}=A z+B\left[F_{r}(z)+u+D_{r}(t, z)\right]
\end{aligned}
$$

with $A=\left[\begin{array}{ccccc}0 & 1 & 0 & \cdots & 0 \\ 0 & 0 & 1 & \cdots & 0 \\ \vdots & \vdots & \vdots & \cdots & \cdots \\ 0 & 0 & 0 & \cdots & \vdots \\ 0 & 0 & 0 & \cdots & 0\end{array}\right], B=\left[\begin{array}{c}0 \\ \vdots \\ 0 \\ 1\end{array}\right]$, where $x=\left[x_{1}, \ldots, x_{n}\right]^{T} \epsilon$ $R^{n}$ and $z=\left[z_{1}, \ldots, z_{n}\right]^{T} \in R^{n}$ are the state vectors of the drive and response systems, respectively. $F_{d}(x)$ and $F_{r}(z)$ are unknown nonlinear smooth functions and $u=\varphi(v)$ is the input nonlinearity, with $v$ being the control input which will be designed later. $D_{d}(t, x)$ and $D_{r}(t, z)$ are the external disturbances of the drive and response systems, respectively.

The input nonlinearity $u=\varphi(v)$ under consideration is given by $[48-50]$

$$
\varphi(v)= \begin{cases}\varphi_{+}(v)\left(v-v_{+}\right), & v>v_{+} \\ 0, & -v_{-} \leq v \leq v_{+} \\ \varphi_{-}(v)\left(v+v_{-}\right), & v<-v_{-}\end{cases}
$$

with $\varphi_{+}(v)>0$ and $\varphi_{-}(v)>0$ being nonlinear smooth functions of $v, v_{+}>0$ and $v_{-}>0$. Note that this model contains both sector nonlinearity and dead-zone. The nonlinearity $\varphi(v)$ also has the following features:

$$
\begin{array}{ll}
\left(v-v_{+}\right) \varphi(v) \geq m_{+}^{*}\left(v-v_{+}\right)^{2}, & v>v_{+} \\
\left(v+v_{-}\right) \varphi(v) \geq m_{-}^{*}\left(v+v_{-}\right)^{2}, & v<-v_{-}
\end{array}
$$

with $m_{+}^{*}$ and $m_{-}^{*}$ being so-called "the gain reduction tolerances" [48-50].

Design Objective. Determine an output-feedback control law $v$ to achieve a projective lag-synchronization between the drive system and the response one, while ensuring that all involved signals in the closed-loop system remain bounded.

To facilitate the control system design, the following usual assumptions are considered and will be used in the subsequent developments.

Assumption 1. The state vectors of the drive and response systems are not measurable, except the system outputs (i.e., except $x_{1}$ and $z_{1}$ ). 
Assumption 2.

(i) The nonlinear functions $\varphi_{+}(v)$ and $\varphi_{-}(v)$ are unknown.

(ii) But, the constants $v_{+}, v_{-}, m_{+}^{*}$, and $m_{-}^{*}$ are assumed to be known.

Assumption 3. The external disturbances, $D_{d}(t, x)$ and $D_{r}(t, z)$, are bounded, respectively, by

$$
\begin{gathered}
\left|D_{d}(t, x)\right| \leq c_{d} \\
\left|D_{r}(t, z)\right| \leq c_{r},
\end{gathered}
$$

where $c_{d}$ and $c_{r}$ are some unknown positive constants.

Definition 4. The drive and response systems (2) are projective lag-synchronized if there exists a scaling factor $\lambda$ such that $e=$ $z-\lambda x(t-\tau) \rightarrow 0$ as $t \rightarrow \infty$, where $\tau>0$ is a constant propagation delay or transmission delay. This means that the transmitted signal is received $\tau$ time late after it was sent. The value of $\tau$ depends on the channel or the distance between drive and response system.

Remark 5. From Definition 4, it is easy to see that, for $\tau=$ 0 , the complete synchronization, antisynchronization, and projective synchronization are the special cases when the scaling factor takes the values $\lambda=+1, \lambda=-1$, and $\lambda \neq 1$ and -1 , respectively. And when $\lambda=1$, one obtains the lagsynchronization.

From (2) and Definition 4, one can write the dynamics of the lag-synchronization error as

$$
\begin{aligned}
\dot{e}= & \dot{z}-\lambda \dot{x}(t-\tau)=A e+B\left[-\lambda F_{d}\left(x_{\tau}\right)-\lambda D_{d}\left(t, x_{\tau}\right)\right. \\
& \left.+F_{r}(z)+u+D_{r}(t, z)\right]=A e+B\left[F_{r}(z)+u+P_{1}\right],
\end{aligned}
$$

where $x_{\tau}=x(t-\tau)$ and

$$
P_{1}=D_{r}(t, z)-\lambda F_{d}\left(x_{\tau}\right)-\lambda D_{d}\left(t, x_{\tau}\right) .
$$

Note that one can easily show the existence of a constant $c_{1}>0$ such as $\left|P_{1}\right| \leq c_{1}$, for the following reasons: $x$ evolves in a compact set (an intrinsic property of the (noncontrolled) chaotic systems), also the delayed state $x_{\tau}$ is bounded and the external disturbances, $D_{d}(t, x)$ and $D_{r}(t, z)$, are already assumed to be bounded, and finally the function $F_{d}\left(x_{\tau}\right)$ is smooth and with a bounded argument.

Since $F_{r}(z)$ is unknown and the vector $e$ is immeasurable, in this paper, one will use

(1) a fuzzy adaptive system to approximate the uncertain functions,

(2) an observer to estimate the projective lagsynchronization error $e$.

\section{Controller Design for Projective Lag-Synchronization}

This section proposes a fuzzy adaptive output-feedback controller for lag-projective synchronization of the driveresponse system (2) using Lyapunov stability theory. The proposed synchronization scheme is shown in Figure 1.

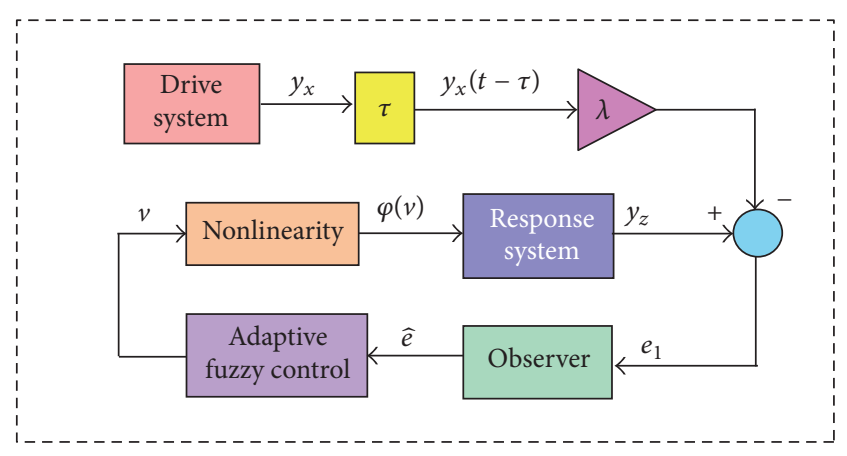

FIGURE 1: Projective lag-synchronization scheme.

One can rewrite the dynamics of the lag-synchronization errors as follows:

$$
\begin{aligned}
\dot{e} & =A e+B\left[F_{r}(e+\lambda x(t-\tau))+u+P_{1}\right] \\
e_{1} & =C e
\end{aligned}
$$

where $C=\left[\begin{array}{llll}1 & 0 & \cdots & 0\end{array}\right]$. Note that the pair $(C, A)$ is observable.

Remark 6.

(1) The controllability property of the pair $(A, B)$ guarantees the existence of a feedback gain vector, $K_{c}$, so that the characteristic polynomial of $A-B K_{c}^{T}$ is strictly Hurwitz.

(2) The observability property of the pair $(C, A)$ ensures the existence of an observer gain vector, $K_{o}$, so that the characteristic polynomial of $A-K_{o} C$ is strictly Hurwitz.

According to fuzzy approximation theorem [28], the unknown function $F_{r}(e+\lambda x(t-\tau))$ can be optimally approximated by a linearly parameterized fuzzy system, as follows [47]:

$$
F_{r}(e+\lambda x(t-\tau))=\theta^{* T} \psi(e)+\varepsilon(e, x(t-\tau))
$$

with $\psi(e)$ being the vector of FBFs (which are assumed to be designed a priori), $\varepsilon(e, x(t-\tau))$ being the fuzzy approximation error, and $\theta^{*}$ being the optimal value of the adjustable parameter vector of the fuzzy system (9) which is defined as

$$
\theta^{*}=\underset{\theta}{\operatorname{argmin}}\left[\sup _{e \in \Omega_{e}}\left|F_{r}(e+\lambda x(t-\tau))-\theta^{T} \psi(e)\right|\right] .
$$

According to [28], the fuzzy approximation error $\varepsilon(e, x(t-\tau))$ is bounded.

Then, (8) becomes

$$
\begin{aligned}
\dot{e} & =A e+B\left[\theta^{* T} \psi(e)+u+P_{2}\right] \\
e_{1} & =C e
\end{aligned}
$$

where $P_{2}=P_{1}+\varepsilon(e, x(t-\tau))$. 
Since the lag-synchronization-error vector $e$ is not available for measurement, one designs the following linear observer to estimate it:

$$
\begin{aligned}
\dot{\hat{e}} & =A_{c} \widehat{e}+K_{o} \widetilde{e}_{1} \\
\hat{e}_{1} & =C \hat{e},
\end{aligned}
$$

where $\widehat{e}$ is the estimate of $e, K_{o}=\left[k_{o 1}, \ldots, k_{o n}\right]^{T} \in R^{n}$ is the gains vector of observer, $A_{c}=A-B K_{c}^{T}$, and $K_{c}=$ $\left[k_{c 1}, \ldots, k_{c n}\right]^{T} \in R^{n}$ is the feedback gain vector.

Now, one defines the observation-error vector as $\widetilde{e}=$ $\left[\widetilde{e}_{1}, \ldots, \widetilde{e}_{n}\right]^{T}=e-\widehat{e}$. From (12) and (11), the dynamics of this observation error can be obtained as follows:

$$
\begin{aligned}
\dot{\tilde{e}} & =A_{o} \widetilde{e}+B\left[\theta^{* T} \psi(e)+\varphi(v)+P_{3}\right] \\
\tilde{e}_{1} & =C \widetilde{e}
\end{aligned}
$$

with $A_{o}=A-K_{o} C$ and

$$
P_{3}=P_{2}+K_{c}^{T} \widehat{e}
$$

Then, we can rewrite (13) using the time-frequency (mixed) notation as follows [51, 52]:

$$
\tilde{e}_{1}=H(s)\left[\theta^{* T} \psi(e)+\varphi(v)+P_{3}\right],
$$

where $s$ is the Laplace variable and $H(s)=C\left(S I-A_{o}\right)^{-1} B$ is the stable transfer function of (13). It is worth noting that this mixed notation is very valuable in the adaptive control literature [51-56]. It also refers to the convolution between the inverse Laplace transform $H(s)$ and the term $\theta^{* T} \psi(e)+$ $\varphi(v)+P_{3}$.

Since $H(s)$ is not SPR, one introduces a low-pass filter $T(s)$ such that $\bar{H}(s)=H(s) T^{-1}(s)$ becomes SPR:

$$
\begin{aligned}
& \tilde{e}_{1} \\
& =\bar{H}(s)\left[\theta^{* T} T(s)[\psi(e)]+T(s)[\varphi(v)]+T(s)\left[P_{3}\right]\right] \\
& =\bar{H}(s)\left[\theta^{* T} \psi(\widehat{e})+\varphi(v)+P_{4}\right]
\end{aligned}
$$

with

$$
\begin{aligned}
P_{4}= & \theta^{* T} T(s)[\psi(e)]+T(s)[\varphi(v)]+T(s)\left[P_{3}\right] \\
& -\theta^{* T} \psi(\widehat{e})-\varphi(v) .
\end{aligned}
$$

Remark 7. $H(s)$ is SPR, with $s=\sigma+j \omega$ if the following conditions are satisfied [57]:

(a) When $s$ is real, $H(s)$ is real.

(b) The poles of $H(s)$ are not in the right half-plane.

(c) For any real $\omega$, the real part of $H(j \omega)$ is positive; that is, $\operatorname{Re}[H(j \omega)] \geq 0$.

Assumption 8. One assumes that $\left|P_{4}\right| \leq k_{p 0}^{*}+k_{p 1}^{*}|v|+$ $k_{p 2}^{*}|T(s)[v]|+k_{p 3}^{*}\left|T(s)\left[K_{c}^{T} \hat{e}\right]\right|=K_{p}^{* T} W$, with $K_{p}^{* T}=$ $\left[k_{p 0}^{*}, k_{p 1}^{*}, k_{p 2}^{*}, k_{p 3}^{*}\right]$ being an unknown positive vector, and $W^{T}=\left[1,|v|,|T(s)[v]|,\left|T(s)\left[K_{c}^{T} \hat{e}\right]\right|\right]$.
Let us define a novel error $e_{m 1}$, called the modified error, as follows:

$$
e_{m 1}=\widetilde{e}_{1}+e_{a 1}
$$

with $e_{a 1}$ being the auxiliary error. Its dynamics are given by

$$
e_{a 1}=\bar{H}(s)\left[-K_{p}^{T} W \tanh \left(\frac{K_{p}^{T} W e_{m 1}}{\varepsilon}\right)\right],
$$

where $K_{p}$ is the estimate of the unknown vector $K_{p}^{*}$ and $\varepsilon>0$ is a small design constant. $\tanh (\cdot)$ designates the usual hyperbolic tangent function.

From (16), (18), and (19), one can obtain

$$
\begin{gathered}
e_{m 1}=\bar{H}(s)\left[\theta^{* T} \psi(\widehat{e})+\varphi(v)+P_{4}\right. \\
\left.-K_{p}^{T} W \tanh \left(\frac{K_{p}^{T} W e_{m 1}}{\varepsilon}\right)\right] .
\end{gathered}
$$

The state-space presentation of (20) can be given by

$$
\begin{aligned}
\dot{e}_{m} & =\bar{A}_{o} e_{m}+\bar{B}\left[\theta^{* T} \psi(\widehat{e})+\varphi(v)+P_{4}\right. \\
& \left.-K_{p}^{T} W \tanh \left(\frac{K_{p}^{T} W e_{m 1}}{\varepsilon}\right)\right] \\
e_{m 1} & =\bar{C} e_{m}
\end{aligned}
$$

where $e_{m}=\left[e_{m 1}, \ldots, e_{m n}\right]^{T}$ and $\left(\bar{A}_{o} \in R^{n \times n}, \bar{B} \in R^{n \times 1}, \bar{C} \in\right.$ $\left.R^{1 \times n}\right)$ is a minimal state realization of $\bar{H}(s)=H(s) T^{-1}(s)=$ $\bar{C}^{T}\left(S I-\bar{A}_{o}\right)^{-1} \bar{B}$ and $\bar{C}=[1,0, \ldots, 0]$.

Since $\bar{H}(s)$ is SPR, the following relation holds:

$$
\begin{gathered}
\bar{A}_{o} P+P \bar{A}_{o}=-Q<0 \\
P \bar{B}=\bar{C}^{T},
\end{gathered}
$$

where $P=P^{T}>0$ and $Q=Q^{T}>0$. Later, expressions (21) and (22) will be exploited in the stability analysis.

To achieve our objective, the control input can be determined as

$$
v= \begin{cases}-\xi \rho \operatorname{sign}\left(e_{m 1}\right)-v_{-}, & e_{m 1}>0 \\ 0, & e_{m 1}=0 \\ -\xi \rho \operatorname{sign}\left(e_{m 1}\right)+v_{+}, & e_{m 1}<0\end{cases}
$$

with $\xi>1 / \eta$, and $\eta=\min \left\{m_{-}^{*}, m_{+}^{*}\right\}$, where

$$
\rho=w_{2}\|\psi(\widehat{e})\|+w_{1},
$$

where $w_{1}$ is a design positive constant and $w_{2}$ is an adaptive parameter estimating the upper bound of $\left\|\theta^{*}\right\|$; that is, $w_{2}^{*} \geq$ $\left\|\theta^{*}\right\|$. 
The adaptive laws for the control law (23) are defined as

$$
\begin{aligned}
& \dot{w}_{2}=-\gamma_{w} \sigma_{w} w_{2}+\gamma_{w}\left|e_{m 1}\right|\|\psi(\widehat{e})\|, \quad \text { with } w_{2}(0)>0 \\
& \dot{K}_{p}=-\gamma_{K} \sigma_{K} K_{p}+\gamma_{K}\left|e_{m 1}\right| W, \quad \text { with } K_{p}(0)>0,
\end{aligned}
$$

where $\gamma_{K}, \sigma_{K}, \gamma_{w}$, and $\sigma_{w}$ are strictly positive design parameters.

Theorem 9. Consider the drive and response systems given by (2) (or (1)) under Assumptions 1-3 and 8. Then, the projective lag-synchronization is realized by using the fuzzy adaptive output-feedback controller (23)-(25) and observer (12).

Proof of Theorem 9. Consider the following Lyapunov function:

$$
V=\frac{1}{2} e_{m}^{T} P e_{m}+\frac{1}{2 \gamma_{K}} \widetilde{K}_{p}^{T} \widetilde{K}_{p}+\frac{1}{2 \gamma_{w}} \widetilde{w}_{2}^{2},
$$

where $\widetilde{K}_{p}=K_{p}-K_{p}^{*}$ and $\widetilde{w}_{2}=w_{2}-w_{2}^{*}$.

The time derivative of $V$ is given as follows:

$$
\dot{V}=\frac{1}{2} e_{m}^{T} P \dot{e}_{m}+\frac{1}{2} \dot{e}_{m}^{T} P e_{m}+\frac{1}{\gamma_{K}} \widetilde{K}_{p}^{T} \dot{K}_{p}+\frac{1}{\gamma_{w}} \widetilde{w}_{2} \dot{w}_{2} .
$$

Evaluating (27) along (21) and (25) and using Assumption 8, one gets

$$
\begin{aligned}
\dot{V} \leq & -\frac{1}{2} e_{m}^{T} Q e_{m}+e_{m 1} \varphi(v)+w_{2}^{*}\left|e_{m 1}\right|\|\psi(\widehat{e})\| \\
& +\left|e_{m 1}\right| K_{p}^{* T} W-K_{p}^{T} W e_{m 1} \tanh \left(\frac{K_{p}^{T} W e_{m 1}}{\varepsilon}\right) \\
& +\frac{1}{\gamma_{K}} \widetilde{K}_{p}^{T} \dot{K}_{p}+\frac{1}{\gamma_{w}} \widetilde{w}_{2} \dot{w}_{2} \\
= & -\frac{1}{2} e_{m}^{T} Q e_{m}+e_{m 1} \varphi(v)+\rho\left|e_{m 1}\right|-w_{1}\left|e_{m 1}\right| \\
& +\left|e_{m 1}\right| K_{p}^{T} W-K_{p}^{T} W \tanh \left(\frac{K_{p}^{T} W e_{m 1}}{\varepsilon}\right) \\
& -\sigma_{K} \widetilde{K}_{p}^{T} K_{p}-\sigma_{w} \widetilde{w}_{2} w_{2} .
\end{aligned}
$$

From (4) and (23), one can easily get the following expressions:

$$
\begin{gathered}
v<-v_{-} \quad \text { for } e_{m 1}>0 \Longrightarrow \\
\left(v+v_{-}\right) \varphi(v) \geq m_{-}^{*}\left(v+v_{-}\right)^{2} \geq \eta\left(v+v_{-}\right)^{2} \\
v>v_{+} \quad \text { for } e_{m 1}<0 \Longrightarrow \\
\left(v-v_{+}\right) \varphi(v) \geq m_{+}^{*}\left(v-v_{+}\right)^{2} \geq \eta\left(v-v_{+}\right)^{2} .
\end{gathered}
$$

Considering (23) again, one can establish

$$
\begin{aligned}
e_{m 1} & >0 \Longrightarrow \\
\left(v+v_{-}\right) \varphi(v) & =-\xi \rho \operatorname{sign}\left(e_{m 1}\right) \varphi(v) \\
& \geq \eta \xi^{2} \rho^{2}\left[\operatorname{sign}\left(e_{m 1}\right)\right]^{2} \\
e_{m 1} & <0 \Longrightarrow \\
\left(v-v_{+}\right) \varphi(v) & =-\xi \rho \operatorname{sign}\left(e_{m 1}\right) \varphi(v) \\
& \geq \eta \xi^{2} \rho^{2}\left[\operatorname{sign}\left(e_{m 1}\right)\right]^{2} .
\end{aligned}
$$

Using the fact that $e_{m 1} \operatorname{sign}\left(e_{m 1}\right)=\left|e_{m 1}\right|$ and while $\rho>0$, one gets for all $e_{m 1}$

$$
e_{m 1} \varphi(v) \leq-\xi \eta \rho\left|e_{m 1}\right|
$$

Substituting (31) into (28), one can obtain

$$
\begin{aligned}
\dot{V} \leq & -\frac{1}{2} e_{m}^{T} Q e_{m}-(\xi \eta-1) \rho\left|e_{m 1}\right|-w_{1}\left|e_{m 1}\right| \\
& +\left|e_{m 1}\right| K_{p}^{T} W-K_{p}^{T} W e_{m 1} \tanh \left(\frac{K_{p}^{T} W e_{m 1}}{\varepsilon}\right) \\
& -\sigma_{K} \widetilde{K}_{p}^{T} K_{p}-\sigma_{w} \widetilde{w}_{2} w_{2} .
\end{aligned}
$$

On the other hand, one can establish that

$$
\begin{aligned}
& -\sigma_{K} \widetilde{K}_{p}^{T} K_{p} \leq-\frac{\sigma_{K}}{2}\left\|\widetilde{K}_{p}\right\|^{2}+\frac{\sigma_{K}}{2}\left\|K_{p}^{*}\right\|^{2} \\
& -\sigma_{w} \widetilde{w}_{2} w_{2} \leq-\frac{\sigma_{w}}{2} \widetilde{w}_{2}+\frac{\sigma_{w}}{2} w_{2}^{* 2} \\
& \left|K_{p}^{T} W e_{m 1}\right|-K_{p}^{T} W e_{m 1} \tanh \left(\frac{K_{p}^{T} W e_{m 1}}{\varepsilon}\right) \leq \bar{\varepsilon} \\
& =0.2783 \varepsilon .
\end{aligned}
$$

By exploiting (33), (32) becomes

$$
\dot{V} \leq-\frac{1}{2} e_{m}^{T} Q e_{m}-\frac{\sigma_{K}}{2}\left\|\widetilde{K}_{p}\right\|^{2}-\frac{\sigma_{w}}{2} \widetilde{w}_{2}^{2}+\pi
$$

where $\pi=\bar{\varepsilon}+\left(\sigma_{K} / 2\right)\left\|K_{p}^{*}\right\|^{2}+\left(\sigma_{w} / 2\right) w_{2}^{* 2}$.

Let $\mu=\min \left\{\lambda_{\min }(Q) / \lambda_{\max }(P), \gamma_{w} \sigma_{w}, \gamma_{K} \sigma_{K}\right\}$; hence one can rewrite (34) as follows:

$$
\dot{V} \leq-\mu V+\pi
$$

where $\lambda_{\text {min }}(X)$ and $\lambda_{\max }(X)$ are the smallest and largest eigenvalues of the matrix $X$, respectively.

(35) can be expressed as follows:

$$
\frac{d\left(V e^{\mu t}\right)}{d t} \leq \pi e^{\mu t} .
$$


And integrating (36) over $[0, t]$ yields

$$
0 \leq V(t) \leq \frac{\pi}{\mu}+\left(V(0)-\frac{\pi}{\mu}\right) e^{-\mu t}
$$

Therefore all signals of the closed-loop system are bounded.

From (26) and (37), one has

$$
\left\|e_{m}\right\| \leq\left(\frac{2}{\lambda_{\min }(P)}\left(\frac{\pi}{\mu}+\left(V(0)-\frac{\pi}{\mu}\right) e^{-\mu t}\right)\right)^{1 / 2},
$$

where $V(0)=(1 / 2) e_{m}^{T}(0) P e_{m}(0)+\left(1 / 2 \gamma_{K}\right) \widetilde{K}_{p}^{T}(0) \widetilde{K}_{p}(0)+$ $\left(1 / 2 \gamma_{w}\right) w_{2}^{2}(0)$.

From (38), one can conclude on the asymptotic convergence of the solution $e_{m}$ to the following bounded region:

$$
\Omega_{e_{m}}=\left\{e_{m} \mid\left\|e_{m}\right\| \leq\left(\frac{2}{\lambda_{\min }(P)} \frac{\pi}{\mu}\right)^{1 / 2}\right\} .
$$

From (18), (19), and (39), one can establish easily the convergence and the boundedness of $e_{a 1}$ and $\widetilde{e}_{1}$.

The proof of this theorem is now completed.

Remark 10. If $v_{+}=v_{-}=v_{0}$, expression (23) can be simply rewritten as

$$
v=-\left(\xi w_{2}\|\psi(\widehat{e})\|+\xi w_{1}+v_{0}\right) \operatorname{sign}\left(e_{m 1}\right) .
$$

In $(40)$, the sign function, that is, $\operatorname{sign}\left(e_{m 1}\right)$, can cause the undesirable chattering phenomenon. In practice, the latter is generally replaced by an equivalent and smooth function (e.g., $\left.\tanh \left(k_{s 1} e_{m 1}\right)\right)$ :

$$
v=-\left(\xi w_{2}\|\psi(\widehat{e})\|+\xi w_{1}+v_{0}\right) \tanh \left(k_{s 1} e_{m 1}\right)
$$

with $k_{s 1}>0$ being a high constant value.

Remark 11. More importantly, the design of a lag-synchronization system based on output-feedback controller for a class of uncertain drive-response systems with input nonlinearities has a major interest in both theory and practice.

(a) Theoretical Interests. Compared to previous works [8-14, 16-18], our theoretical contributions are the following:

(1) Design of a projective lag-synchronization system by considering the ubiquitous input nonlinearities (i.e., sector nonlinearities and dead-zone), the uncertain dynamics of both models, and the immeasurability of the states of drive-response system is theoretically challenge. To the best of authors' knowledge, the projective lag-synchronization for this class of driveresponse systems with all these features has rarely been studied in the literature.

(2) The proposed fuzzy adaptive output-feedback control requires the so-called SPR condition on the lagsynchronization errors. It should be noted that the design of an output-feedback controller dealing with input nonlinearities (particularly, sector nonlinearity and dead-zone) and by using a SPR approach is not theoretically simple. This is why in the literature there are few fundamental results dealing with this control problem.

(b) Practical Interests. The proposed synchronization approach has the following practical interests:

(1) The proposed projective lag-synchronization approach is characterized by one scalar transmitted signal. This feature is of practical significant importance.

(2) The effect of ubiquitous input nonlinearities (sector nonlinearities and dead-zone) has been taken into account in the stability analysis and the design of the control system. In practice, it is well known that the nonconsideration of the latter may lead to a serious degradation of the system's performances and even cause system instability.

(3) In particular, this projective lag-synchronization approach has also a prospective application in secure communication due to its safety against attack and unmasking.

\section{Illustrative Simulation Examples}

Three academic examples are provided in this section to validate the effectiveness of this proposed synchronization approach.

Example 1. Consider the projective lag-synchronization between chaotic Gyros system and Duffing oscillator.

The Drive System (Chaotic Gyros System) [58]

$$
\begin{aligned}
\dot{x}_{1}= & x_{2} \\
\dot{x}_{2}= & -\alpha^{2} \frac{\left(1-\cos \left(x_{1}\right)\right)^{2}}{\sin ^{3}\left(x_{1}\right)}-c_{1} x_{2}-c_{2} x_{2}^{2} \\
& +\left(\beta+f \sin \left(\omega_{x} t\right)\right) \sin \left(x_{1}\right)+D_{d}(t, x),
\end{aligned}
$$

where $=\left[\begin{array}{ll}x_{1} & x_{2}\end{array}\right]^{T}, \alpha^{2}=100, c_{1}=0.5, c_{2}=0.05, \beta=1$, $\omega_{x}=2$, and $f=35.5 . D_{d}(t, x)$ is assumed to be a normally (Gaussian) distributed random signal with a variance $=0.5$ and a mean $=0.5$.

The Response System (Duffing Oscillator) [59]

$$
\begin{aligned}
\dot{z}_{1}= & z_{2} \\
\dot{z}_{2}= & -p_{1} z_{2}-p_{2} z_{1}-p_{3} z_{1}^{3}+q \sin \left(\omega_{z} t\right)+u \\
& +D_{r}(t, z),
\end{aligned}
$$

where $z=\left[\begin{array}{ll}z_{1} & z_{2}\end{array}\right]^{T}, p_{1}=0.4, p_{2}=-1.1, p_{3}=1, q=2.1$, $\omega_{z}=1.8$, and $D_{r}(t, z)=\sin (6 t)$. 
Then, this chaotic drive-response system can be rewritten as follows:

$$
\begin{aligned}
\dot{x} & =A x+B\left(F_{d}(x)+D_{d}(t, x)\right) \\
y_{x} & =x_{1}=C x \\
\dot{z} & =A z+B\left(F_{r}(z)+u+D_{r}(t, z)\right), \\
y_{z} & =z_{1}=C z,
\end{aligned}
$$

where $A=\left[\begin{array}{ll}0 & 1 \\ 0 & 0\end{array}\right], B=\left[\begin{array}{l}0 \\ 1\end{array}\right]$, and $C^{T}=\left[\begin{array}{l}1 \\ 0\end{array}\right] \cdot u=\varphi(v)$ is the input nonlinearity which is defined below, and $v$ is the control input to be designed.

The input nonlinearities $\varphi(v)$ are assumed to be described by $[34,48]$

$$
\begin{aligned}
u= & \varphi(v) \\
= & \begin{cases}(v-0.5)\left(1.5-0.3 e^{0.3|\sin (v)|}\right) & v>0.5 \\
0 & -0.5 \leq v \leq 0.5 \\
(v+0.5)\left(1.5-0.3 e^{0.3|\sin (v)|}\right) & v<-0.5 .\end{cases}
\end{aligned}
$$

To estimate the synchronization error, the following linear observer is designed:

$$
\begin{aligned}
\dot{\hat{e}} & =A_{c} \widehat{e}+K_{o}\left(y_{x}(t-\tau)-y_{z}-\widehat{e}_{1}\right) \\
\widehat{e}_{1} & =C \widehat{e}
\end{aligned}
$$

with $\widehat{e}=\left[\widehat{e}_{1}, \widehat{e}_{2}\right]^{T}$ being the estimate of $e=\left[e_{1}, e_{2}\right]^{T}, K_{o}=$ $\left[2 \alpha, \alpha^{2}\right]^{T}$ being the observer gain vector with $\alpha=80, A_{c}=$ $A-B K_{c}^{T}$, and $K_{c}=[90,60]^{T}$.

Based on Theorem 9 and Remark 10, the control for system (44) can be designed as (40) or (41) with adaptive laws (25). Its associated design parameters are chosen as follows: $\tau=0.5 \mathrm{sec}, \lambda=1, w_{1}=100, \varepsilon=0.2, \gamma_{w}=100, \sigma_{w}=0.001$, $\gamma_{k}=100$, and $\sigma_{k}=0.001$. For each variable of the entries of the designed fuzzy system, as in $[47,60]$, one defines three membership functions (one triangular and two trapezoidal) uniformly distributed on the following intervals: $\left[\begin{array}{ll}-2 & 2\end{array}\right]$ for $\widehat{e}_{1}$ and $\left[\begin{array}{ll}-2 & 2\end{array}\right]$ for $\hat{e}_{2}$.

One selects the SPR filter $T(s)$ so that $\bar{H}(s)=$ $H(s) T^{-1}(s)=\left(1 /\left(s^{2}+160 s+6400\right)\right) T^{-1}(s)$ is SPR, as follows:

$$
T(s)=\frac{1}{0.3906 s+11.7721} .
$$

From the expression of $\bar{H}(s)$, one can find that $\bar{A}=\left[\begin{array}{ll}-2 \alpha & 1 \\ -\alpha^{2} & 0\end{array}\right]$, $\bar{B}^{T}=\left[\begin{array}{ll}0.3906 & 11.7721\end{array}\right]$, and $\bar{C}^{T}=\left[\begin{array}{ll}1 & 0\end{array}\right]$.

By choosing $Q_{1}=\left[\begin{array}{cc}30 & 3 \\ 3 & 0.5\end{array}\right]$ and solving (22), one gets

$$
P_{1}=\left[\begin{array}{cc}
10.0937 & -0.2500 \\
-0.2500 & 0.0083
\end{array}\right] \text {. }
$$

The initial conditions are chosen as $x(0)=\left[x_{1}(0), x_{2}(0)\right]^{T}=$ $[-1,1]^{T}, z(0)=\left[z_{1}(0), z_{2}(0)\right]^{T}=[0.5,2]^{T}, w_{2}(0)=10$, and $K_{p}(0)=[0.01,0.01,0.01,0.01]^{T}$.
Note that, because $v_{+}=v_{-}=v_{0}=0.5$, the variablestructure controller (23) can be directly replaced by (40). Two cases are considered to show the validity of the proposed controller.

(a) Simulation by Using the Discontinuous Controller (40). Figure 2 shows that the proposed controller performs well. In fact, one can see from Figures 2(a) and 2(b) that the states of response system $\left(z_{1}, z_{2}\right)$ effectively track that of the drive system $\left(\lambda x_{1}(t-\tau), \lambda x_{2}(t-\tau)\right)$, despite the presence of the immeasurable states, uncertain dynamics, dead-zone at the input, and external disturbances. From Figure 2(c), it is clear also that the estimates of the synchronization errors are bounded and asymptotically converge towards small values. The corresponding control signal is bounded and not smooth in Figure 2(d).

(b) Simulation by Using the Smooth Controller (41). Figure 3 provides the simulation results. From Figures 3(a) and 3(b), one can observe that the states of the response system $\left(z_{1}, z_{2}\right)$ effectively follow the corresponding desired trajectories $\left(\lambda x_{1}(t-\tau), \lambda x_{2}(t-\tau)\right)$. From Figure 3(c), one can see that the estimates of the synchronization errors are wellbounded and converge to a small value. In Figure 3(d), the control signal is smooth, bounded, and admissible.

Example 2. Now, we will consider the projective lagsynchronization between two uncertain similar chaotic systems of the third order.

The Drive System (Genesio Chaotic System) [61]

$$
\begin{aligned}
& \dot{x}_{1}=x_{2} \\
& \dot{x}_{2}=x_{3} \\
& \dot{x}_{3}=-6 x_{1}-2.92 x_{2}-1.2 x_{3}+x_{1}^{2}+D_{d}(t, x),
\end{aligned}
$$

where $x=\left[x_{1}, x_{2}, x_{3}\right]^{T}$ and $D_{d}(t, x)$ is assumed to be a normally (Gaussian) distributed random signal with a variance $=0.5$ and a mean $=0.5$.

The Response System (Genesio Chaotic System) [61]

$$
\begin{aligned}
& \dot{z}_{1}=z_{2} \\
& \dot{z}_{2}=z_{3} \\
& \dot{z}_{3}=-6 z_{1}-2.92 z_{2}-1.2 z_{3}+z_{1}^{2}+u+D_{r}(t, z),
\end{aligned}
$$

where $D_{r}(t, z)=\sin (6 t)$ and $z=\left[z_{1}, z_{2}, z_{3}\right]^{T}$ is the state vector of the response system.

Then, this chaotic drive-response system can be rewritten as follows:

$$
\begin{aligned}
\dot{x} & =A x+B\left(F_{d}(x)+D_{d}(t, x)\right), \\
y_{x} & =x_{1}=C x \\
\dot{z} & =A z+B\left(F_{r}(z)+u+D_{r}(t, z)\right), \\
y_{z} & =z_{1}=C z,
\end{aligned}
$$




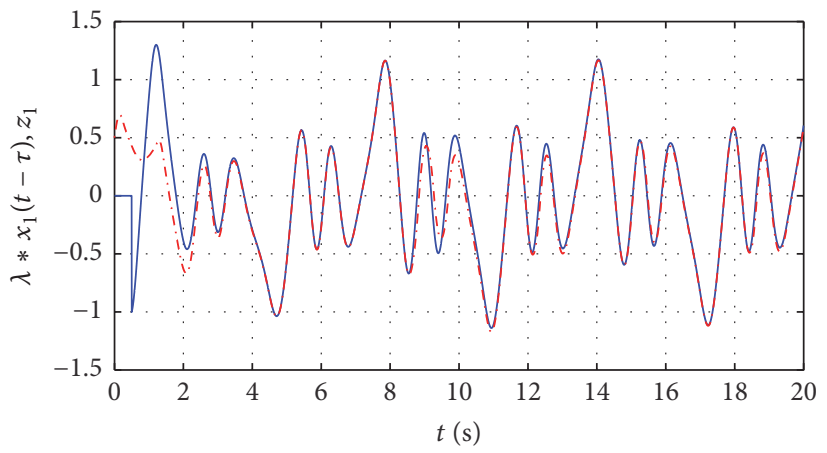

(a)

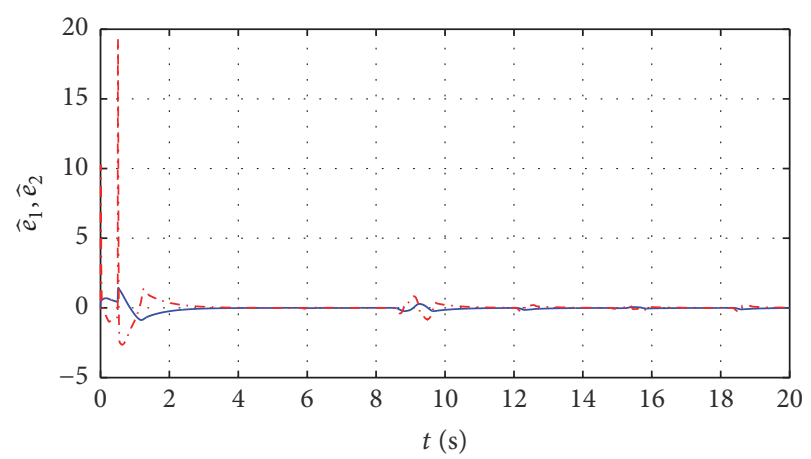

(c)

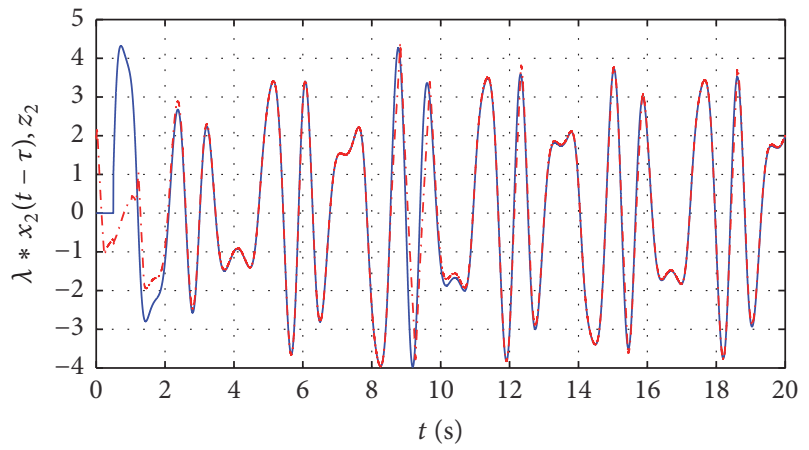

(b)

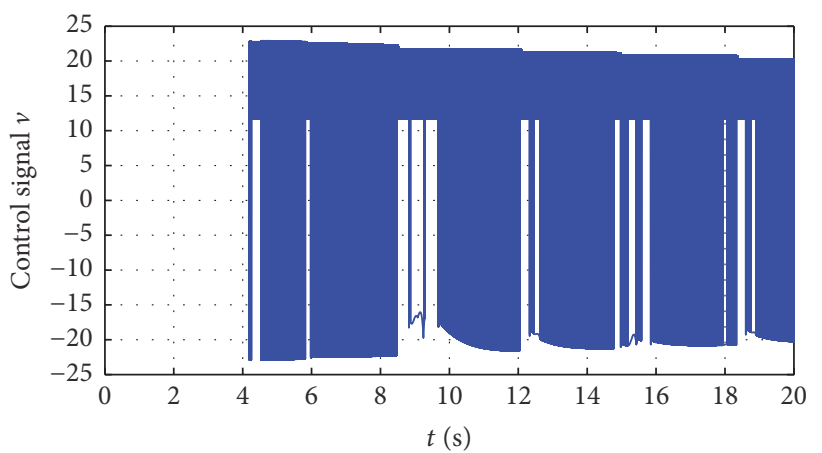

(d)

FIGURE 2: Simulation results (for Example 1, in case 1): (a) states: $\lambda x_{1}(t-\tau)$ (solid line) and $z_{1}$ (dash-dot line). (b) States: $\lambda x_{2}(t-\tau)$ (solid line) and $z_{2}$ (dash-dot line). (c) Estimates of the synchronization errors $\widehat{e}_{1}$ (solid line) and $\widehat{e}_{2}$ (dash-dot line). (d) Control signal $v$.

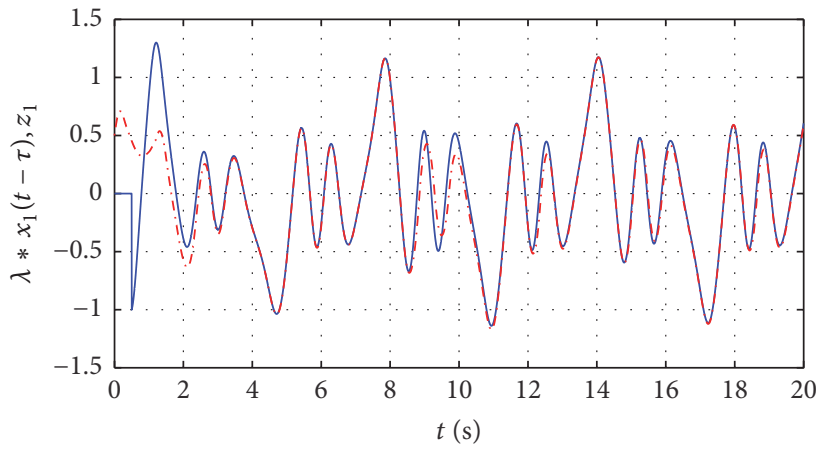

(a)

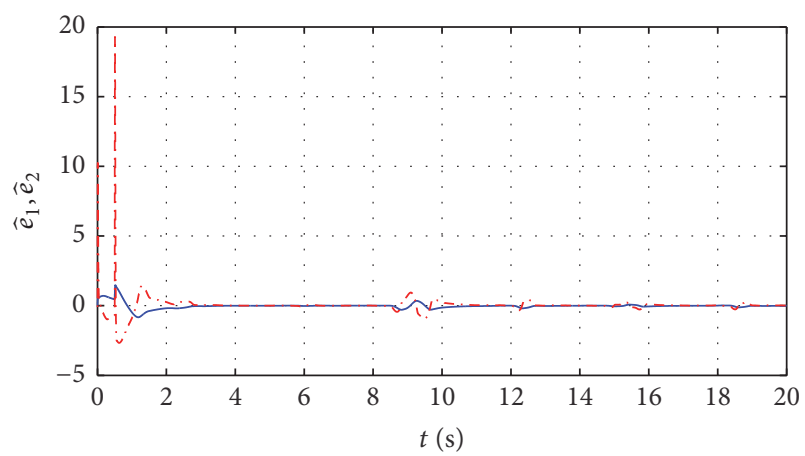

(c)

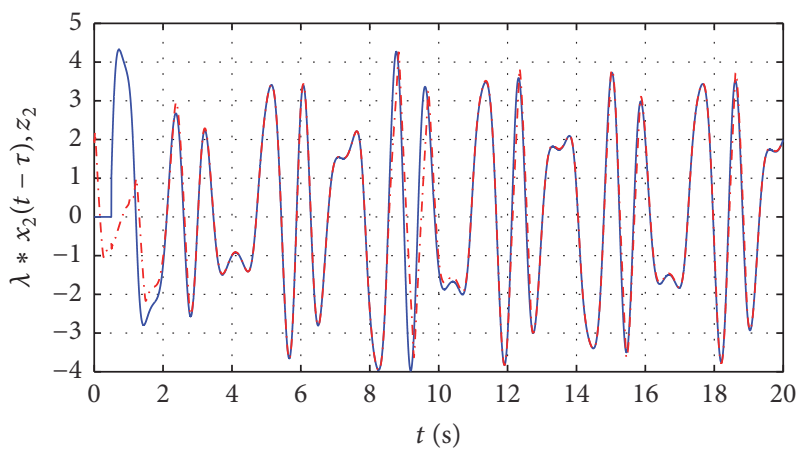

(b)

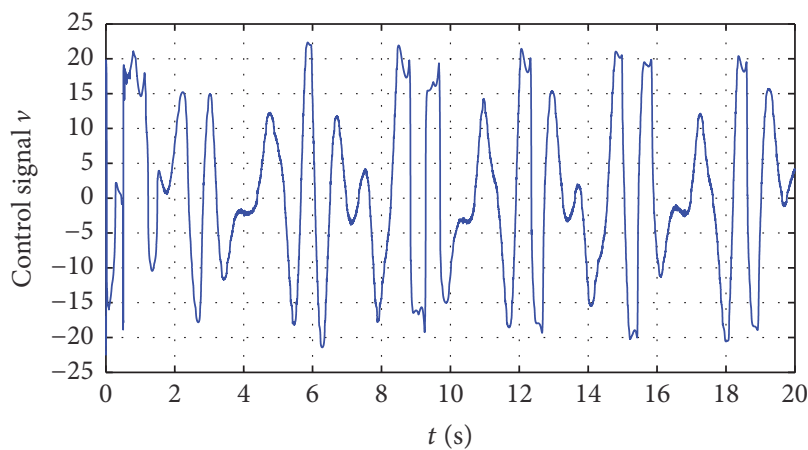

(d)

FIGURE 3: Simulation results (for Example 1, in case 2): (a) states: $\lambda x_{1}(t-\tau)$ (solid line) and $z_{1}$ (dash-dot line). (b) States: $\lambda x_{2}(t-\tau)$ (solid line) and $z_{2}$ (dash-dot line). (c) Estimates of the synchronization errors $\widehat{e}_{1}$ (solid line) and $\widehat{e}_{2}$ (dash-dot line). (d) Control signal $v$. 


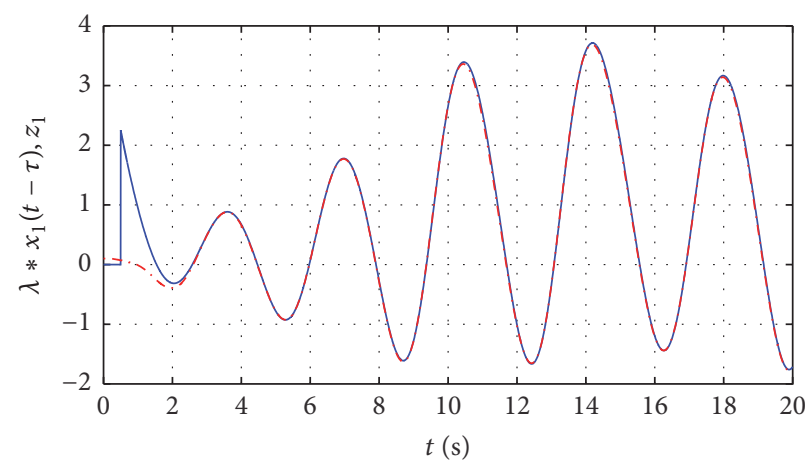

(a)

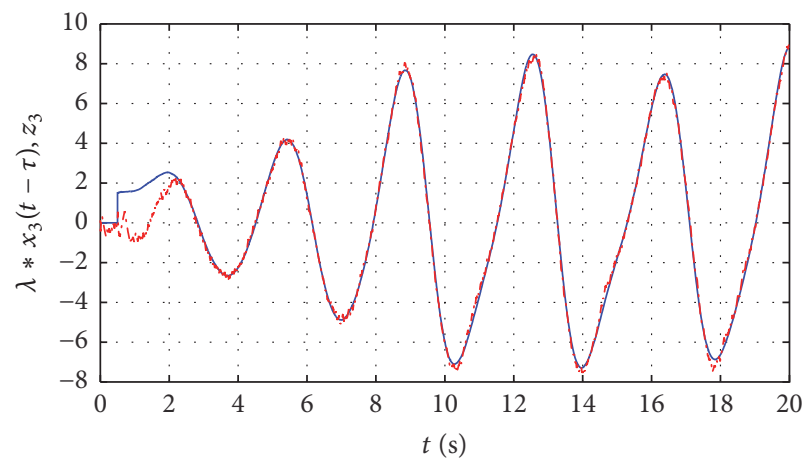

(c)

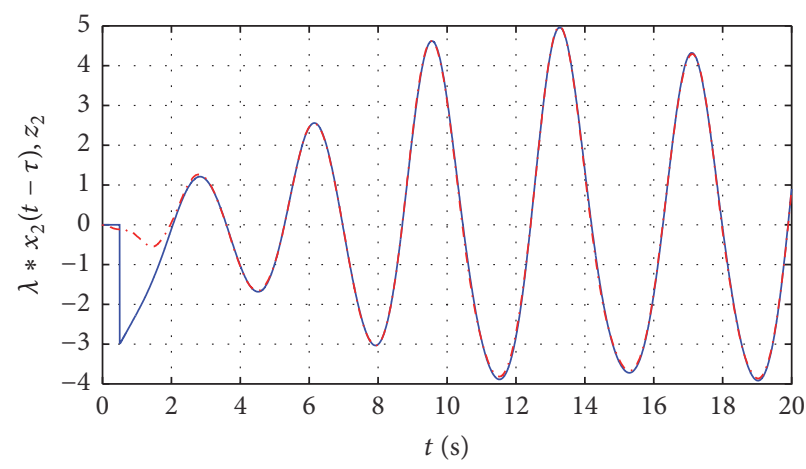

(b)

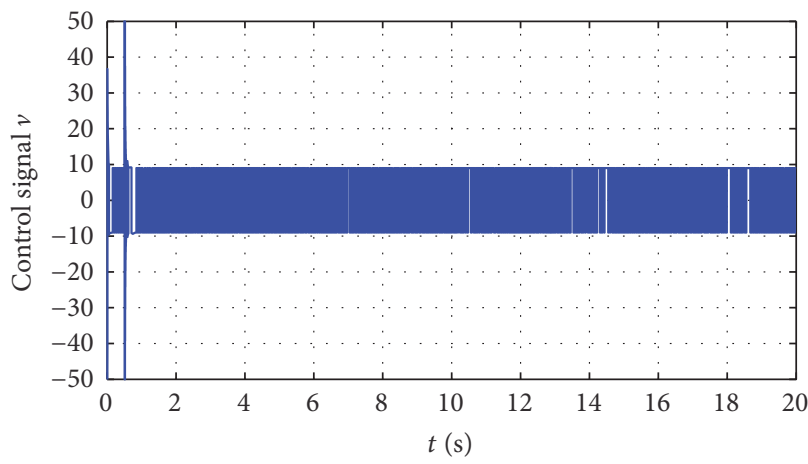

(d)

Figure 4: Simulation results (for Example 2): (a) states: $\lambda x_{1}(t-\tau)$ (solid line) and $z_{1}$ (dash-dot line). (b) States: $\lambda x_{2}(t-\tau)$ (solid line) and $z_{2}$ (dash-dot line). (c) States: $\lambda x_{3}(t-\tau)$ (solid line) and $z_{3}$ (dash-dot line). (d) Control signal $v$.

where $A=\left[\begin{array}{lll}0 & 1 & 0 \\ 0 & 0 & 1 \\ 0 & 0 & 0\end{array}\right], B=\left[\begin{array}{l}0 \\ 0 \\ 1\end{array}\right]$, and $C=\left[\begin{array}{lll}1 & 0 & 0\end{array}\right] . u=\varphi(v)$ is the input nonlinearity which is defined below, and $v$ is the control input to be designed.

The input nonlinearities $\varphi(v)$ are assumed to be described by $[34,48]$

$$
\begin{aligned}
u & =\varphi(v) \\
& = \begin{cases}(v-1)\left(1.5-0.3 e^{0.3|\sin (v)|}\right) & v>1 \\
0 & -1 \leq v \leq 1 \\
(v+1)\left(1.5-0.3 e^{0.3|\sin (v)|}\right) & v<-1 .\end{cases}
\end{aligned}
$$

To estimate the synchronization error, the following linear observer is designed:

$$
\begin{aligned}
\dot{\hat{e}} & =A_{c} \widehat{e}+K_{o}\left(y_{x}(t-\tau)-y_{z}-\widehat{e}_{1}\right) \\
\hat{e}_{1} & =C \widehat{e}
\end{aligned}
$$

with $\widehat{e}=\left[\widehat{e}_{1}, \widehat{e}_{2}, \widehat{e}_{3}\right]^{T}$ being the estimate of $e=\left[e_{1}, e_{2}, e_{3}\right]^{T}$, $K_{o}=\left[3 \alpha, 3 \alpha^{2}, \alpha^{3}\right]^{T}$ being the observer gain vector with $\alpha=$ $60, A_{c}=A-B K_{c}^{T}$, and $K_{c}=[64,48,12]^{T}$.

Based on Theorem 9 and Remark 10, the controller for system (51) can be designed as (40) with adaptive laws (25). Its associated design parameters are chosen as follows: $\tau=$ $0.5 \mathrm{sec}, \lambda=0.75, w_{1}=1, \varepsilon=0.2, \gamma_{w}=1000, \sigma_{w}=0.01$, $\gamma_{k}=100$, and $\sigma_{k}=2$. For each input of the fuzzy system, as in
$[47,60]$, one designs three membership functions (the central membership function is triangular and, however, the two others are trapezoidal) uniformly distributed on the following intervals: $\left[\begin{array}{ll}-5 & 5\end{array}\right]$ for $\widehat{e}_{1},\left[\begin{array}{ll}-5 & 5\end{array}\right]$ for $\widehat{e}_{2}$, and $\left[\begin{array}{ll}-10 & 10\end{array}\right]$ for $\widehat{e}_{3}$.

We select the SPR filter $T(s)$ so that $\bar{H}(s)=H(s) T^{-1}(s)=$ $\left(1 /\left(s^{3}+180 s^{2}+10800 S+216000\right)\right) T^{-1}(s)$ is SPR, as follows: $T(s)=1 /\left(0.0003 s^{2}+0.0120 S+0.2726\right)$.

From the expression of $\bar{H}(s)$, one can find that $\bar{A}=$ $\left[\begin{array}{ccc}-3 \alpha & 1 & 0 \\ -3 \alpha^{2} & 0 & 1 \\ -\alpha^{3} & 0 & 0\end{array}\right], \bar{B}^{T}=\left[\begin{array}{lll}0.0003 & 0.0120 & 0.2726\end{array}\right]$, and $\bar{C}^{T}=$ $\left[\begin{array}{lll}1 & 0 & 0\end{array}\right]$.

By choosing $Q=\left[\begin{array}{rrr}30 & 3 & 3 \\ 3 & 3 & 1 \\ 3 & 1 & 1\end{array}\right]$ and solving (22), one gets

$$
P=\left[\begin{array}{ccc}
14985 & -2 & -12 \\
-2 & 11 & -1 \\
-12 & -1 & 0.0001
\end{array}\right]
$$

The initial conditions are chosen as $x(0)=\left[x_{1}(0), x_{2}(0)\right.$, $\left.x_{3}(0)\right]^{T}=[3,-4,2]^{T}, z(0)=\left[z_{1}(0), z_{2}(0), z_{3}(0)\right]^{T}=[0.1$, $0.0,0.1]^{T}, w_{2}(0)=40$, and $K_{p}(0)=[0,0,0,0]^{T}$.

The projective lag-synchronization response of system (51) is presented in Figure 4. It is obvious from the latter that the trajectories of response system $\left(z_{1}, z_{2}, z_{3}\right)$ effectively track that of the drive system $\left(\lambda x_{1}(t-\tau), \lambda x_{2}(t-\tau), \lambda x_{3}(t-\tau)\right)$, despite the presence of the immeasurable states of chaotic systems, uncertain dynamics, dead-zone at the input, and 


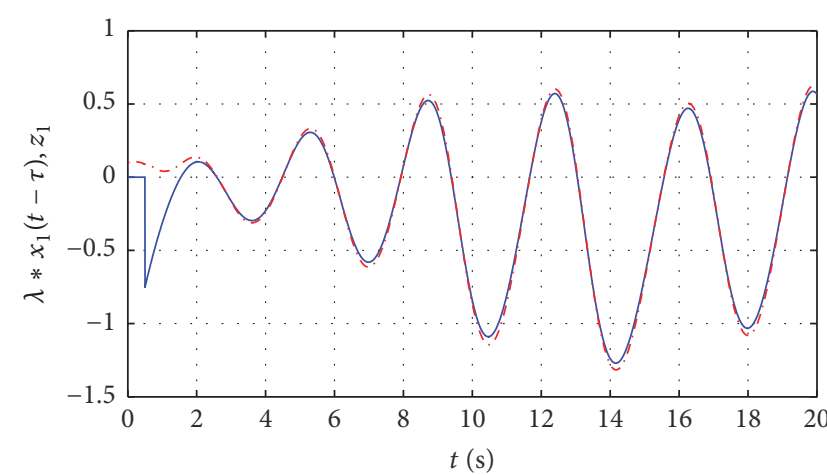

(a)

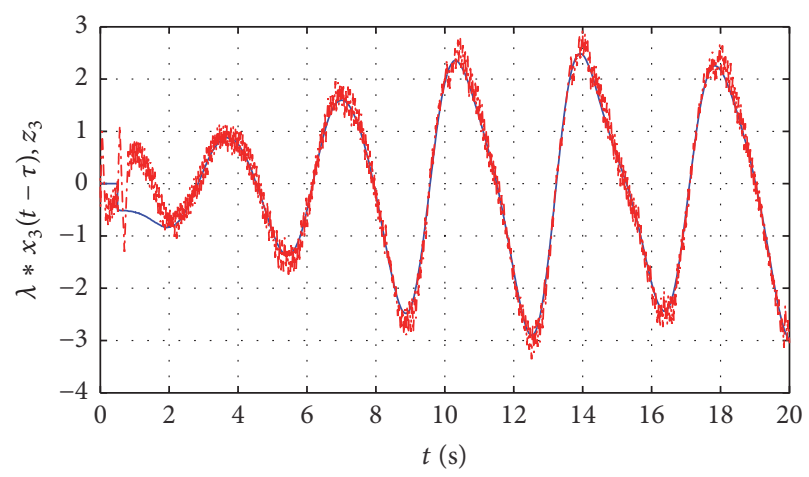

(c)

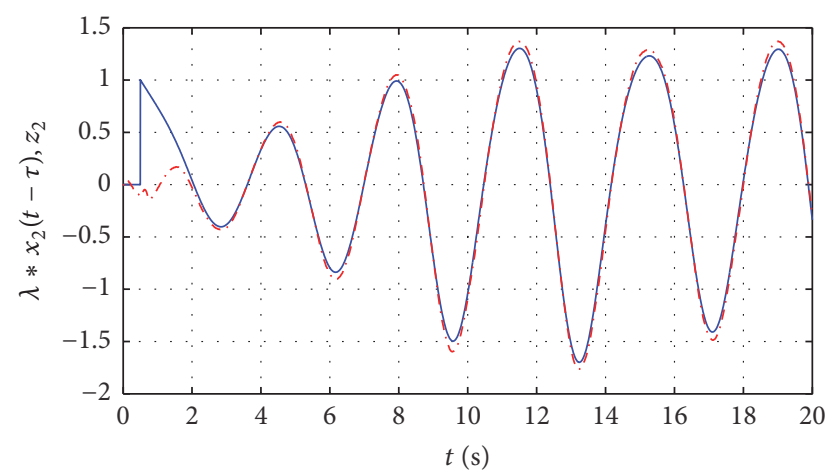

(b)

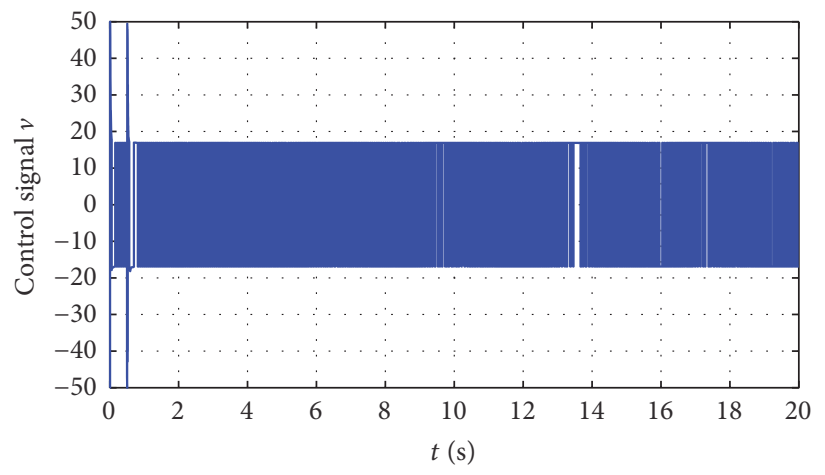

(d)

Figure 5: Simulation results (for Example 3): (a) states: $\lambda x_{1}(t-\tau)$ (solid line) and $z_{1}$ (dash-dot line). (b) States: $\lambda x_{2}(t-\tau)$ (solid line) and $z_{2}$ (dash-dot line). (c) States: $\lambda x_{3}(t-\tau)$ (solid line) and $z_{3}$ (dash-dot line). (d) Control signal $v$.

external disturbances. The corresponding control signal is bounded.

Example 3. We consider now the projective lag-synchronization between two different chaotic systems of the third order.

The Drive System (Genesio Chaotic System)

$$
\begin{aligned}
& \dot{x}_{1}=x_{2} \\
& \dot{x}_{2}=x_{3} \\
& \dot{x}_{3}=-6 x_{1}-2.92 x_{2}-1.2 x_{3}+x_{1}^{2}+D_{d}(t, x),
\end{aligned}
$$

where $x=\left[x_{1}, x_{2}, x_{3}\right]^{T}$ and $D_{d}(t, x)$ is taken as a normally (Gaussian) distributed random signal with a variance $=0.5$ and a mean $=0.5$.

The Response System (Arneodo Chaotic System) [62]

$$
\begin{aligned}
& \dot{z}_{1}=z_{2} \\
& \dot{z}_{2}=z_{3} \\
& \dot{z}_{3}=5.5 z_{1}-3.5 z_{2}-z_{3}-z_{1}^{3}+u+D_{r}(t, z),
\end{aligned}
$$

where $D_{r}(t, z)=\sin (6 t)$ and $z=\left[z_{1}, z_{2}, z_{3}\right]^{T}$.

Note that this synchronization scheme is designed as that of the second example, except that the response system is selected as an Arneodo chaotic system and $\lambda=-0.25$.
Therefore, the fuzzy system and the synchronization-error observer are designed as in Example 2, and the initial conditions and the design parameters are also selected as in Example 2.

Figure 5 provides the simulation results of this example, from which it can be clearly seen that the trajectories of response system $\left(z_{1}, z_{2}, z_{3}\right)$ effectively track the trajectories of the drive system $\left(\lambda x_{1}(t-\tau), \lambda x_{2}(t-\tau), \lambda x_{3}(t-\tau)\right)$, despite the presence of immeasurable states of chaotic systems, uncertain dynamics, input nonlinearities, and external disturbances.

\section{Conclusion}

The problem of adaptive fuzzy output-feedback controlbased projective lag-synchronization for unknown driveresponse (or master-slave) chaotic systems has been investigated in this paper. In the design process, the input nonlinearities (dead-zone together with sector nonlinearities) have been considered. To effectively handle the unknown functions in the drive-response system, fuzzy adaptive systems have been incorporated in the control system. To deal with the input nonlinearities, the proposed controller has been designed in a variable-structure framework. And to estimate the synchronization-error states, a simple linear observer has been constructed. Finally, three academic examples have been given to demonstrate the effectiveness of the proposed lagsynchronization approach. In our future work, the investigation for chaotic fractional-order drive-response systems 
subject to unavailable states and more nonsmooth input nonlinearities deserves further research.

\section{Competing Interests}

The authors declare that there is no conflict of interests regarding the publication of this paper.

\section{References}

[1] S. Bowong, M. Kakmeni, and R. Koina, "Chaos synchronization and duration time of a class of uncertain chaotic systems," Mathematics and Computers in Simulation, vol. 71, no. 3, pp. 212-228, 2006

[2] C. Wang, Y. He, J. Ma, and L. Huang, "Parameters estimation, mixed synchronization, and antisynchronization in chaotic systems," Complexity, vol. 20, no. 1, pp. 64-73, 2014.

[3] Y.-W. Wang and Z.-H. Guan, "Generalized synchronization of continuous chaotic system," Chaos, Solitons and Fractals, vol. 27, no. 1, pp. 97-101, 2006.

[4] J. Yan and C. Li, "Generalized projective synchronization of a unified chaotic system," Chaos, Solitons and Fractals, vol. 26, no. 4, pp. 1119-1124, 2005.

[5] G.-H. Li, "Projective synchronization of chaotic system using backstepping control," Chaos, Solitons and Fractals, vol. 29, no. 2, pp. 490-494, 2006.

[6] G.-H. Li, "Generalized projective synchronization between Lorenz system and Chen's system," Chaos, Solitons and Fractals, vol. 32, no. 4, pp. 1454-1458, 2007.

[7] C. Chen, G. Feng, and X. Guan, "An adaptive lagsynchronization method for time-delay chaotic systems," in Proceedings of the American Control Conference (ACC '05), pp. 4277-4282, June 2005.

[8] S. Pourdehi, D. Karimipour, and P. Karimaghaee, "Outputfeedback lag-synchronization of time-delayed chaotic systems in the presence of external disturbances subjected to input nonlinearity," Chaos, vol. 21, no. 4, Article ID 043128, 2011.

[9] E. M. Shahverdiev, S. Sivaprakasam, and K. A. Shore, "Lag synchronization in time-delayed systems," Physics Letters, Section A: General, Atomic and Solid State Physics, vol. 292, no. 6, pp. 320-324, 2002.

[10] C. Chen, G. Feng, and X. Guan, "An adaptive lagsynchronization method for time-delay chaotic systems," in Proceedings of the American Control Conference, pp. 42774282, Portland, Ore, USA, June 2005.

[11] Y. Chen, X. Chen, and S. Gu, "Lag synchronization of structurally nonequivalent chaotic systems with time delays," Nonlinear Analysis. Theory, Methods \& Applications, vol. 66, no. 9, pp. 1929-1937, 2007.

[12] C. Li, X. Liao, and K.-W. Wong, "Lag synchronization of hyperchaos with application to secure communications," Chaos, Solitons \& Fractals, vol. 23, no. 1, pp. 183-193, 2005.

[13] L. Wang, Z. Yuan, X. Chen, and Z. Zhou, "Lag synchronization of chaotic systems with parameter mismatches," Communications in Nonlinear Science and Numerical Simulation, vol. 16, no. 2, pp. 987-992, 2011.

[14] Y. Xu, W. Zhou, J. Fang, and W. Sun, "Adaptive lag synchronization and parameters adaptive lag identification of chaotic systems," Physics Letters A, vol. 374, no. 34, pp. 3441-3446, 2010.
[15] L. M. Pecora and T. L. Carroll, "Synchronization in chaotic systems," Physical Review Letters, vol. 64, no. 8, pp. 821-824, 1990.

[16] M. Zhao, R. Liu, and Y. Gao, "Dissipative lag synchronization of chaotic Lur'e systems with unknown disturbances," IMA Journal of Mathematical Control and Information, 2015.

[17] G. Wei-Dong, S. Zhi-Yong, W. Xiao-Ming, and Y. Chang-Bin, "Adaptive lag synchronization of uncertain dynamical systems with time delays via simple transmission lag feedback," Chinese Physics B, vol. 22, no. 8, Article ID 080507, 2013.

[18] Z. Sun, "Lag projective synchronization of two chaotic systems with different fractional orders," Journal of the Korean Physical Society, vol. 66, no. 8, pp. 1192-1199, 2015.

[19] A. Khan and M. Shahzad, "Synchronization of circular restricted three body problem with Lorenz hyper chaotic system using a robust adaptive sliding mode controller," Complexity, vol. 18, no. 6, pp. 58-64, 2013.

[20] M. Roopaei, B. R. Sahraei, and T.-C. Lin, "Adaptive sliding mode control in a novel class of chaotic systems," Communications in Nonlinear Science and Numerical Simulation, vol. 15, no. 12, pp. 4158-4170, 2010

[21] I. Ahmad, A. B. Saaban, A. B. Ibrahim, and M. Shahzad, "Global chaos synchronization of new chaotic system using linear active control," Complexity, vol. 21, no. 1, pp. 379-386, 2015.

[22] I. Ahmad, A. Saaban, A. Ibrahim, and M. Shahzad, "A research on the synchronization of two novel chaotic systems based on a nonlinear active control algorithm," Engineering, Technology \& Applied Science Research, vol. 5, no. 1, pp. 739-747, 2015.

[23] J. A. Vargas, E. Grzeidak, and E. Hemerly, "Robust adaptive synchronization of a hyperchaotic finance system," Nonlinear Dynamics, vol. 80, no. 1, pp. 239-248, 2015.

[24] I. Ahmad, A. B. Saaban, A. B. Ibrahim, S. Al-Hadhrami, M. Shahzad, and S. H. Al-Mahrouqi, "A research on adaptive control to stabilize and synchronize a hyperchaotic system with uncertain parameters," An International Journal of Optimization and Control: Theories \& Applications, vol. 5, no. 2, pp. 51-62, 2015.

[25] T.-C. Lin, T.-Y. Lee, and V. E. Balas, "Adaptive fuzzy sliding mode control for synchronization of uncertain fractional order chaotic systems," Chaos, Solitons and Fractals, vol. 44, no. 10, pp. 791-801, 2011.

[26] E. Hwang, C. Hyun, E. Kim, and M. Park, "Fuzzy model based adaptive synchronization of uncertain chaotic systems: robust tracking control approach," Physics Letters A, vol. 373, no. 22, pp. 1935-1939, 2009.

[27] J. Wang, L. Chen, and B. Deng, "Synchronization of Ghostburster neuron in external electrical stimulation via $H_{\infty}$ variable universe fuzzy adaptive control," Chaos, Solitons \& Fractals, vol. 39, no. 5, pp. 2076-2085, 2009.

[28] L. Wang, Adaptive Fuzzy Systems and Control: Design and Stability Analysis, Prentice-Hall, Englewood Cliffs, NJ, USA, 1994.

[29] A. Boulkroune, H. Chekireb, M. Tadjine, and S. Bouatmane, "Observer-based adaptive feedback controller of a class of chaotic systems," International Journal of Bifurcation and Chaos in Applied Sciences and Engineering, vol. 16, no. 11, pp. 3411-3419, 2006.

[30] A. Boulkroune, M. Chekireb, M. Tadjine, and S. Bouatmane, "An adaptive feedback controller with observer for linearizable chaotic systems," Control and Intelligent Systems, vol. 35, no. 2, pp. 162-168, 2007. 
[31] A. Boulkroune and M. M'saad, "A practical projective synchronization approach for uncertain chaotic systems with dead-zone input," Communications in Nonlinear Science and Numerical Simulation, vol. 16, no. 11, pp. 4487-4500, 2011.

[32] A. Boulkroune and M. M'saad, "Fuzzy adaptive observer-based projective synchronization for nonlinear systems with input nonlinearity," Journal of Vibration and Control, vol. 18, no. 3, pp. 437-450, 2012.

[33] S. Hamel and A. Boulkroune, "A generalized function projective synchronization scheme for uncertain chaotic systems subject to input nonlinearities," International Journal of General Systems, vol. 45, no. 6, pp. 689-710, 2016.

[34] S. Hamel, A. Boulkroune, and A. Bouzeriba, "Function vector synchronization based on fuzzy control for uncertain chaotic systems with dead-zone nonlinearities," Complexity, vol. 21, no. 1, pp. 234-249, 2015.

[35] A. Boulkroune and M. M'Saad, "A fuzzy adaptive variablestructure control scheme for uncertain chaotic MIMO systems with sector nonlinearities and dead-zones," Expert Systems with Applications, vol. 38, no. 12, pp. 14744-14750, 2011.

[36] S. Pourdehi and P. Karimaghaee, "Simple adaptive outputfeedback lag-synchronization of multiple time-delayed chaotic systems," Chaos, vol. 22, no. 2, Article ID 023145, 2012.

[37] W. M. Bessa, A. S. de Paula, and M. A. Savi, "Sliding mode control with adaptive fuzzy dead-zone compensation for uncertain chaotic systems," Nonlinear Dynamics, vol. 70, no. 3, pp. 19892001, 2012.

[38] M. P. Aghababa and H. Aghababa, "A novel finite-time sliding mode controller for synchronization of chaotic systems with input nonlinearity," Arabian Journal for Science and Engineering, vol. 38, no. 11, pp. 3221-3232, 2013.

[39] Z. Zhang, J. Lu, L. Gao, and H. Shao, "Exponential synchronization of Genesio-Tesi chaotic systems with partially known uncertainties and completely unknown dead-zone nonlinearity," Journal of the Franklin Institute, vol. 350, no. 2, pp. 347-357, 2013.

[40] Y.-G. Leu, T.-T. Lee, and W.-Y. Wang, "Observer-based adaptive fuzzy-neural control for unknown nonlinear dynamical systems," IEEE Transactions on Systems, Man, and Cybernetics, Part B: Cybernetics, vol. 29, no. 5, pp. 583-591, 1999.

[41] Y.-G. Leu, W.-Y. Wang, and T.-T. Lee, "Observer-based direct adaptive fuzzy-neural control for nonaffine nonlinear systems," IEEE Transactions on Neural Networks, vol. 16, no. 4, pp. 853861, 2005.

[42] H.-X. Li and S. Tong, "A hybrid adaptive fuzzy control for a class of nonlinear MIMO systems," IEEE Transactions on Fuzzy Systems, vol. 11, no. 1, pp. 24-34, 2003.

[43] S. Tong, H.-X. Li, and W. Wang, "Observer-based adaptive fuzzy control for SISO nonlinear systems," Fuzzy Sets and Systems, vol. 148, no. 3, pp. 355-376, 2004.

[44] W.-Y. Wang, Y.-G. Leu, and T.-T. Lee, “Output-feedback control of nonlinear systems using direct adaptive fuzzy-neural controller," Fuzzy Sets and Systems, vol. 140, no. 2, pp. 341-358, 2003.

[45] Y.-J. Liu, S.-C. Tong, W. Wang, and Y.-M. Li, "Observerbased direct adaptive fuzzy control of uncertain nonlinear systems and its applications," International Journal of Control, Automation and Systems, vol. 7, no. 4, pp. 681-690, 2009.

[46] H. F. Ho, Y. K. Wong, A. B. Rad, and W. L. Lo, "State observer based indirect adaptive fuzzy tracking control," Simulation Modelling Practice and Theory, vol. 13, no. 7, pp. 646-663, 2005.
[47] A. Boulkroune, M. Tadjine, M. M'Saad, and M. Farza, "How to design a fuzzy adaptive controller based on observers for uncertain affine nonlinear systems," Fuzzy Sets and Systems, vol. 159, no. 8, pp. 926-948, 2008.

[48] K.-K. Shyu, W.-J. Liu, and K.-C. Hsu, "Design of large-scale time-delayed systems with dead-zone input via variable structure control," Automatica, vol. 41, no. 7, pp. 1239-1246, 2005.

[49] A. Boulkroune, M. M'Saad, and M. Farza, "Adaptive fuzzy controller for multivariable nonlinear state time-varying delay systems subject to input nonlinearities," Fuzzy Sets and Systems, vol. 164, pp. 45-65, 2011.

[50] A. Boulkroune, M. Msaad, and M. Farza, "State and output feedback fuzzy variable structure controllers for multivariable nonlinear systems subject to input nonlinearities," International Journal of Advanced Manufacturing Technology, vol. 71, no. 1-4, pp. 539-556, 2014.

[51] A. Boulkroune, N. Bounar, M. M'saad, and M. Farza, "Indirect adaptive fuzzy control scheme based on observer for nonlinear systems: a novel SPR-filter approach," Neurocomputing, vol. 135, pp. 378-387, 2014.

[52] A. Boulkroune and M. M'saad, "On the design of observerbased fuzzy adaptive controller for nonlinear systems with unknown control gain sign," Fuzzy Sets and Systems, vol. 201, pp. 71-85, 2012.

[53] J. E. Slotine and W. Li, Applied Nonlinear Control, Prentice-Hall, Englewood Cliffs, NJ, USA, 1991.

[54] M. French and E. Rogers, "Approximate models for adaptive feedback linearization," International Journal of Control, vol. 68, no. 6, pp. 1305-1321, 1997.

[55] N. Kim and A. J. Calise, "Several extensions in methods for adaptive output feedback control," IEEE Transactions on Neural Networks, vol. 18, no. 2, pp. 482-494, 2007.

[56] S. S. Sastry and A. Isidori, "Adaptive control of linearizable systems," Institute of Electrical and Electronics Engineers, vol. 34, no. 11, pp. 1123-1131, 1989.

[57] S. Sastry and M. Bodson, Adaptive Control: Stability, Convergence and Robustness, Prentice-Hall, Englewood Cliffs, NJ, USA, 1989.

[58] M. Roopaei, M. Zolghadri Jahromi, R. John, and T.-C. Lin, "Unknown nonlinear chaotic gyros synchronization using adaptive fuzzy sliding mode control with unknown dead-zone input," Communications in Nonlinear Science and Numerical Simulation, vol. 15, no. 9, pp. 2536-2545, 2010.

[59] G. R. Chen and X. Dong, "On feedback control of chaotic continuous-time systems," IEEE Transactions on Circuits and Systems I: Fundamental Theory and Applications, vol. 40, no. 9, pp. 591-601, 1993.

[60] R.-E. Precup and M. L. Tomescu, "Stable fuzzy logic control of a general class of chaotic systems," Neural Computing and Applications, vol. 26, no. 3, pp. 541-550, 2015.

[61] H.-T. Yau and C.-L. Chen, "Chattering-free fuzzy sliding-mode control strategy for uncertain chaotic systems," Chaos, Solitons and Fractals, vol. 30, no. 3, pp. 709-718, 2006.

[62] M. M. Al-sawalha and M. S. M. Noorani, "Anti-synchronization of chaotic systems with uncertain parameters via adaptive control," Physics Letters A, vol. 373, no. 32, pp. 2852-2857, 2009. 


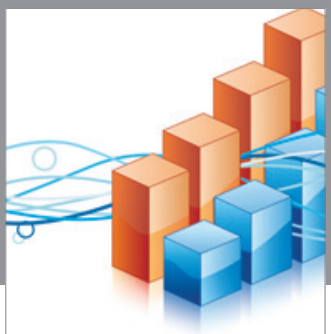

Advances in

Operations Research

vatem alat4

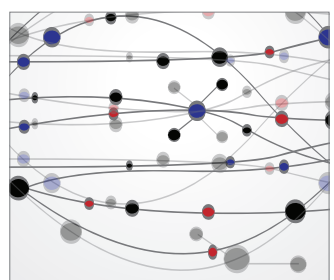

\section{The Scientific} World Journal
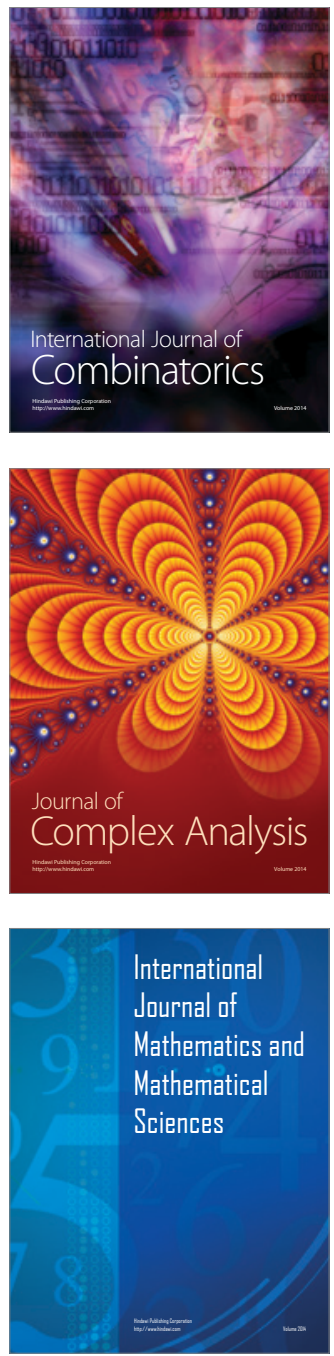
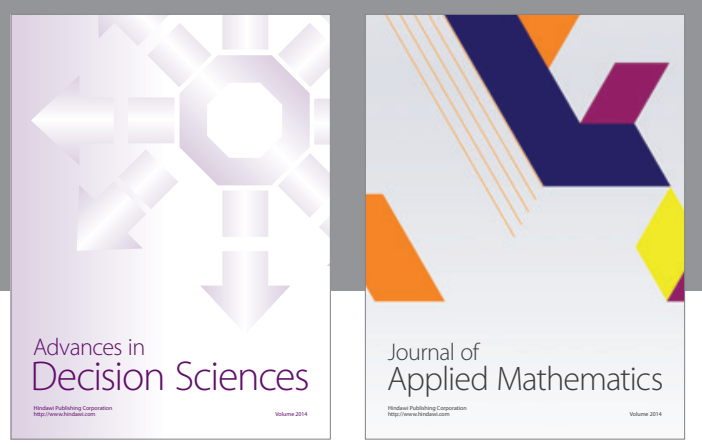

Algebra

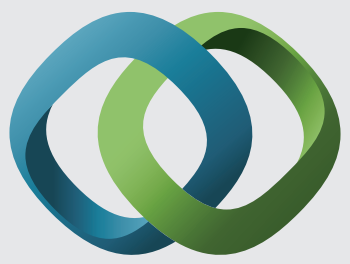

\section{Hindawi}

Submit your manuscripts at

https://www.hindawi.com
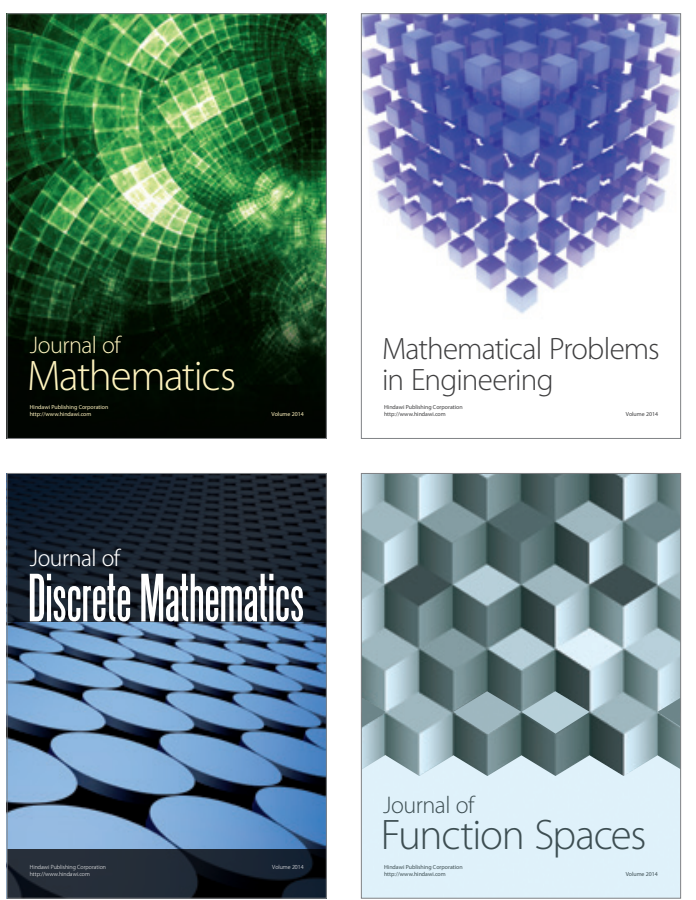

Mathematical Problems in Engineering
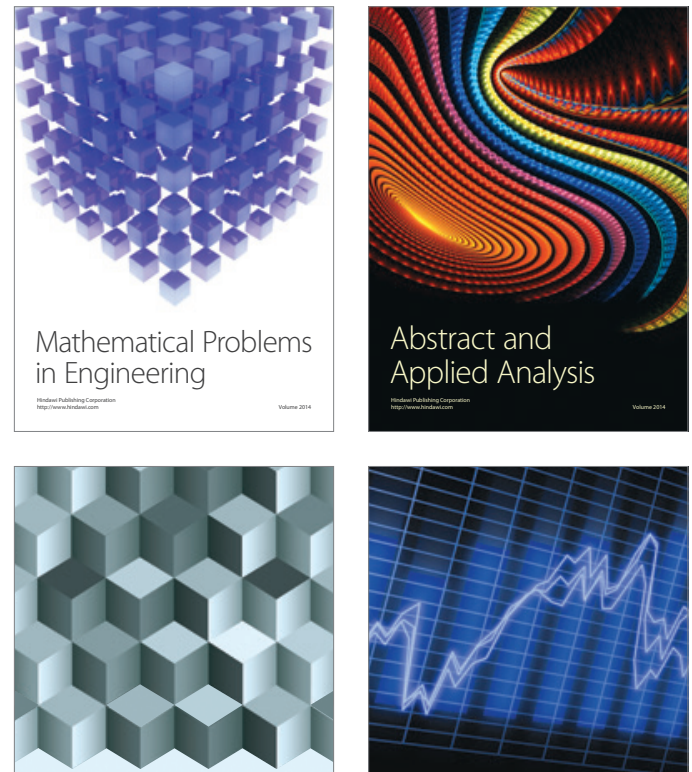

Journal of

Function Spaces

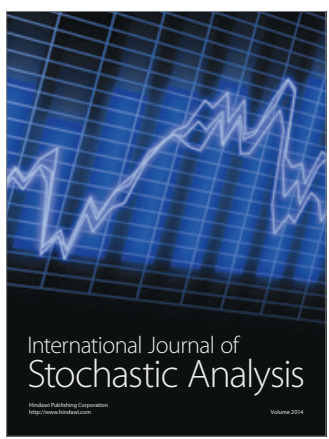

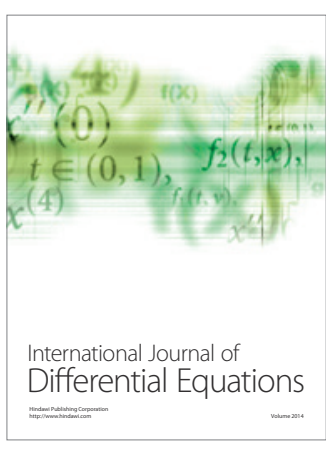
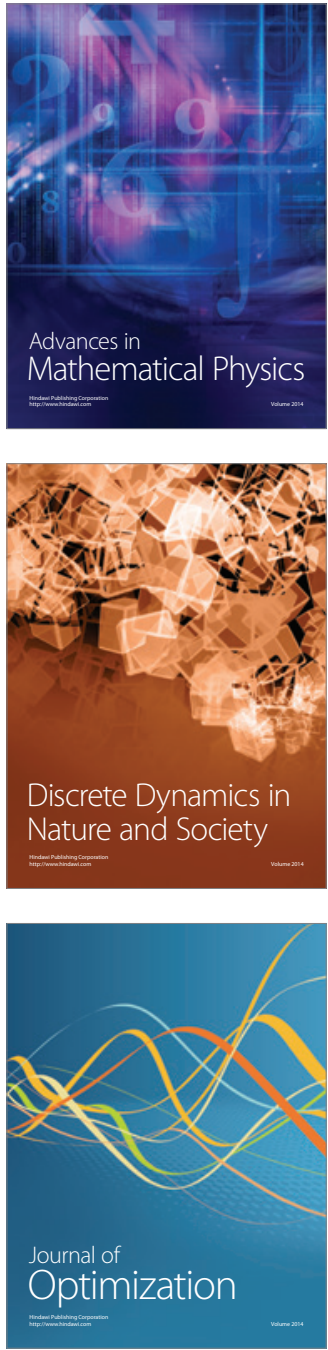